\title{
Identifying the impact of rotation, anisotropy, and energetic particle physics in tokamaks
}

\author{
M.J. Hole ${ }^{1}$, G. von Nessi ${ }^{1}$, M. Fitzgerald ${ }^{1}$, K. \\ McClements $^{2}$, J. Svensson ${ }^{3}$ and the MAST team ${ }^{2}$ \\ ${ }^{1}$ Research School of Physical Sciences and Engineering, Australian National \\ University, Acton 0200, ACT Australia \\ 2 EURATOM/CCFE Fusion Assoc., Culham Science Centre,Abingdon, Oxon \\ OX14 3DB, UK \\ ${ }^{3}$ Max Planck Institute for Plasma Physics, Teilinstitut Greifswald, Germany
}

\begin{abstract}
In this paper we study the effects of poloidal and toroidal rotation, and anisotropy in tokamaks. To resolve these effects from uncertainties in the data, we introduce a Bayesian inference framework which calculates the magnetic configuration probabilistically using Motional Stark Effect and magnetic data. Drawing on these calculations, we compute the poloidal and toroidal Mach numbers in MAST for a discharge with good rotation data. Our calculations confirm that the poloidal Mach number $M_{s, \theta}=v_{\theta} / v_{i} \times B / B_{\theta}$ is near zero (with $v_{\theta}$ and $v_{i}$ the poloidal and thermal velocity, respectively), even on the outboard side where the scaling of poloidal field strength $B_{\theta}$ to total field $B$ is large. In contrast, the toroidal rotation of this plasma reaches a Mach number of 0.5 on axis. The impact of the toroidal rotation on the equilibrium reconstructions of the plasma is however small: it acts to increase the radius of the magnetic axis by $\approx 1 \%$, and lower the central safety factor by $\approx 5 \%$. In comparison, corrections to make the pressure profile consistent with internal measurements such as charge exchange recombination spectroscopy and Thomson scattering have a much larger impact. In other work we compute the level of anisotropy from a TRANSP simulation of a neutral beam heated MAST discharge. This shows a large level of anisotropy, with $p_{\perp} / p_{\|} \approx 1.7$, sufficient to boost the central safety factor by $15 \%$. For this discharge, which is representative of many MAST discharges, the effect of anisotropy and consistent pressure profiles is more pronounced than the toroidal rotation of the plasma.
\end{abstract}

PACS numbers: $52.55 . \mathrm{Fa}, 52.55 .-\mathrm{s}$

Submitted to: Plasma Phys. Control. Fusion 


\section{Introduction}

Due to the increase in plasma external heating, high performance magnetically confined fusion plasmas have drifted far from the simple picture of ideal magnetohydrodynamics (MHD), which describes the plasma as a single, stationary, isotropic Maxwellian fluid. Several important observed deviations include temperature anisotropy, substantial rotation, and significant stored energy residing in the energetic particle population produced by charge exchange of fast beams with thermals.[1] Despite these observations, stationary Grad-Shafranov solvers are still the code of choice for tokamaks.

Poloidal and toroidal flows in tokamak plasmas are of great interest, primarily because they are known to affect both neoclassical [2, 3, 4] and turbulent [5, 6] transport, and also magnetohydrodynamic (MHD) stability [7, 8]. A strong correlation has been observed between highly-sheared poloidal flows and internal transport barriers in several tokamaks, notably in the Joint European Torus (JET) [9]. One of the challenges created by the presence of strong flows is that the traditional approach to modelling plasma equilibria, based on the assumption that inertial terms in the MHD force balance equation can be neglected, is no longer adequate. The Grad-Shafranov (G-S) equation, which describes axis-symmetric equilibria, can be readily generalized to include both toroidal and poloidal flows when it is supplemented with an energy equation, which is required to provide closure. In many analyses of this problem (see McClements and Hole [10] and references therein) an isentropic energy equation has been employed, i.e. plasma entropy per unit mass has been assumed to be constant on a flux surface. While this assumption may be reasonable for some collision-dominated astrophysical plasmas, it is not generally appropriate for present-day tokamaks, in which the collisionality is sufficiently low that electron and ion temperatures are much more likely than specific entropy to be well-approximated by flux functions.

In recent work McClements and Hole [10, 11] have studied the elliptic to hyperbolic "transonic" poloidal flow transition of the flow modified Grad Shafranov equation for tight aspect ratio, and for an isothermal closure condition rather than adiabatic closure condition. They showed that the range of poloidal flows for which the G-S equation is hyperbolic increases with plasma beta and $B_{\theta} / B$, the ratio of the poloidal to total magnetic field, thereby complicating the problem of determining spherical tokamak plasma equilibria with transonic poloidal flows. It was also shown that the calculation of the hyperbolicity criterion can be easily modified when the assumption of isentropic flux surfaces is replaced with the more tokamak-relevant one of isothermal flux surfaces. On the basis of the latter assumption, a simple expression was obtained for the variation of density on a flux surface when poloidal and toroidal flows are simultaneously present. In this work we apply this expression to MAST.

The broader purpose of this paper is to discuss some recent advances in equilibrium modelling and validation, and identify the degree to which rotation, anisotropy and energetic particles modify the configuration. We span several topics: development of a Bayesian inference validation framework for the probabilistic calculation of magnetic configurations, the calculation of Mach numbers from rotation data, magnetics and Motional Stark Effect in MAST spherical tokamak plasmas, the calculation of the impact of anisotropy in MAST plasmas, and toroidally-symmetric equilibrium models which resolve flow and anisotropy. We mention, but leave to future work, calculation of the equilibrium of MAST plasmas with multiple fluids [12], which represent different energetic component of the distribution function, with ions paired 
with electrons within each fluid to ensure quasi-neutrality. Qualitatively, the effect of such multiple fluids on the plasma is similar to comensurate modifications with the thermal profile, although quantitative differences arise due to the balance of energetic and thermal terms. An illustrative MAST-like multi-fluid equilibrium with separate thermal and beam fluids was presented recently by Hole et al [11].

The paper is structured as follows: Sec. 2 introduces a Bayesian inference framework and uses it to compute a magnetic configuration for a neutral beam heated MAST plasma. Section 3 explores rotation-induced asymmetry of plasma profiles, computes the poloidal and toroidal Mach numbers and elucidates the impact of this rotation on the configuration. Section 4 computes the magnitude and impact of anisotropy on a MAST plasma, and finally, Sec. 5 contains concluding remarks.

\section{A Bayesian inference framework to compute magnetic configurations}

Recently, a new integrated data-modelling approach for inference of fusion plasma parameters has emerged which offers a natural framework with which to resolve different physics models. The Bayesian approach to inference in fusion plasmas, developed by multiple authors, $[13,14,15,16,17,18,19,20]$ involves the specification of an initial prior probability distribution function (pdf), $P(\mathcal{I})$, for the vector of plasma parameters, $\mathcal{I}$, which is then updated by taking into account information that the measurements provide through the likelihood pdf $P(\mathcal{D} \mid \mathcal{I})$, where $\mathcal{D}$ is the measurement vector, and the notation $\mathcal{D} \mid \mathcal{I}$ denotes a forward diagnostic model describing the response data $\mathcal{D}$ to plasma parameters $\mathcal{I}$. The result is the posterior distribution $P(\mathcal{I} \mid \mathcal{D})$, the conditional probability assigned after the relevant evidence $\mathcal{D}$ is taken into account, given by Bayes' formula

$$
P(\mathcal{I} \mid \mathcal{D})=P(\mathcal{D} \mid \mathcal{I}) P(\mathcal{I}) / P(\mathcal{D}) .
$$

The advantage of the Bayesian approach over traditional inversion techniques is twofold: (i) prior knowledge, including known parameter inter-dependencies is made explicit, and (ii) as the formulation is probabilistic, random errors, systematic uncertainties and instrumental bias are integral part of the analysis rather than an afterthought.

We have implemented Bayesian inversion using the MINERVA framework. [21] In this framework, probabilistic graphical models are used to project the dependence of the posterior distribution function onto the prior, the data, and the likelihood. An advantage of this approach is that it visualises the complex interdependency between data and model, and thus expedites model development. In this paper we draw on MINERVA to compute the magnetic configuration and its uncertainty of a beam heated discharge. Here, the plasma parameters $\mathcal{I}$ take the vector of toroidal current beams across the plasma cross-section, and the vector $\mathcal{D}$ represents the magnetic pickup coils, poloidal flux loops and the polarisation angle of emitted light from neutrally excited species during beam injection. The prior distribution $P(\mathcal{I})$ of the current beams is a conditional Autoregressive prior, providing some spatially localised correlation between adjacent current beams. Each individual current beam is assumed to have a zero mean normal distribution. Further details of toroidal current tomography of MINERVA on MAST are available elsewhere. [22, 23, 24]

Recently, both MAST neutral beam injectors have been upgraded to $3.8 \mathrm{MW}$. This has enabled plasma performance to be routinely lifted above $\beta_{n} \approx 5$, which was reported in 2005. [1] MAST is also equipped with an array of precision diagnostics 
[25], including a high spatial resolution, single-time point, ruby Thomson Scattering (TS)system, a multi-time point Nd:YAG TS system with a coarser spatial resolution, motional Stark effect (MSE), charge exchange recombination, and fast magnetics. [26]

We have chosen discharge \#20662 for analysis, as poloidal and toroidal rotation profiles are available [27]. Discharge \#20662 was a deuterium plasma with a plasma current of $I_{p}=700 \mathrm{kA}$ in a double-null configuration, which was heated with $1.8 \mathrm{MW}$ of neutral beams at $65 \mathrm{keV}$ injection energy. Figure 1 shows a Bayesian inference of poloidal flux surfaces from MAST discharge \#20662 at $230 \mathrm{~ms}$ using pickup coils, flux loops and MSE data. The time selected corresponds to the high spatial-resolution ruby TS laser, for which the plasma was in L-mode confinement. The figure shows a contour plot of $\psi(R, Z)$ which is calculated from the maximum of the posterior of the distribution of toroidal current beams. Overlaid on the contours are traces of the poloidal field coil cross sections and conducting surface cross sections for the MAST experiment, as well as the last closed flux surface, or plasma boundary, calculated from the plasma beam model and the corresponding EFIT plasma boundary. The main reason for the discrepancy between the EFIT plasma boundary and our Bayesian approach is the inclusion of MSE measurements in the Bayesian treatment: these strongly influence the placement of the plasma boundary on the outboard edge of the plasma.

One outcome of the Bayesian approach is the generation of pdfs of inferred quantities from which the uncertainty can be computed. For instance, Fig. 2 shows the profile of the inferred mean and standard deviation of the toroidal current density for \#20662 at 230ms, as well as the corresponding safety factor or $q$ profile and its uncertainty. Within the body of the plasma (up to a normalised $\psi_{n} \approx 0.97$, with $\psi_{n}=0$ the core and $\psi_{n}=1$ the edge), the uncertainty in $q$ is less than $10 \%$. The large edge uncertainty in the $q$ profile in Fig. 2(b) occurs because while the toroidal current passes to zero at the edge, the error in the toroidal current remains approximately constant at $1.7 \mathrm{kA} \mathrm{m}^{-2}$. Thus, the corresponding uncertainty in the vanishing poloidal field produces a large uncertainty in $q$. At zero poloidal field $q$ and its uncertainty are infinite.

\section{Poloidal and toroidal rotation}

The value of computing the magnetic configuration probabilistically is that it provides an uncertainty measure, which can be propagated to compute the uncertainty of inferred quantities dependent on the magnetic configuration. One such quantity is the poloidal Mach number, which is dependent on the ratio $B / B_{\theta}$, with $B$ the local field strength and $B_{\theta}$ the local poloidal field strength.

Our treatment of flowing tokamak equilibria is based on the framework of ideal MHD [28] with the poloidal Mach number below unity. When toroidal and poloidal flows $v_{\phi}, v_{\theta}$ are both present there exists a flux function $\Omega$ such that

$$
\Omega=\frac{v_{\phi}}{R}-\frac{v_{\theta}}{R} \frac{B_{\phi}}{B_{\theta}},
$$

and the Bernoulli relation for isothermal poloidal flux surfaces can be written as

$$
H(\psi)=\frac{2 T(\psi)}{m_{i}} \ln \left(\frac{\rho}{\rho_{0}}\right)+\frac{v_{\phi}^{2}+v_{\theta}^{2}}{2}-\Omega R v_{\phi},
$$




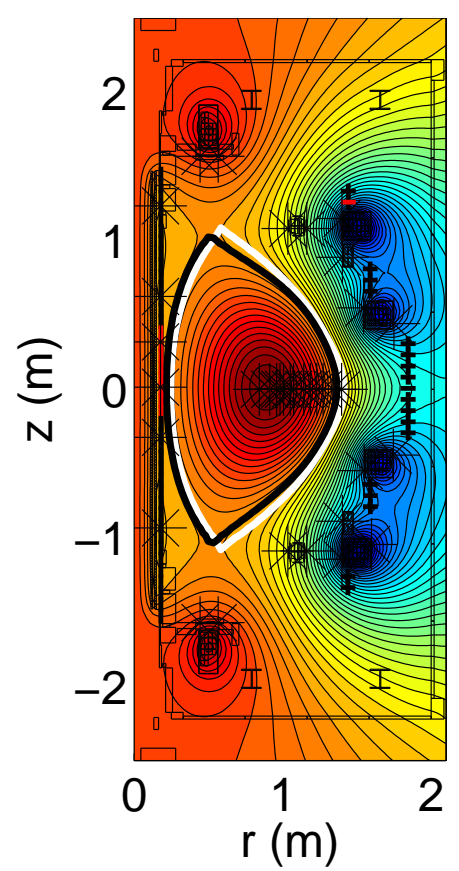

Figure 1. Poloidal flux surfaces inferred for MAST shot \#20662 at $230 \mathrm{~ms}$ using pickup coils, flux loops and MSE. The last closed flux surface from the plasma beam model / EFIT is plotted in heavy black/white.
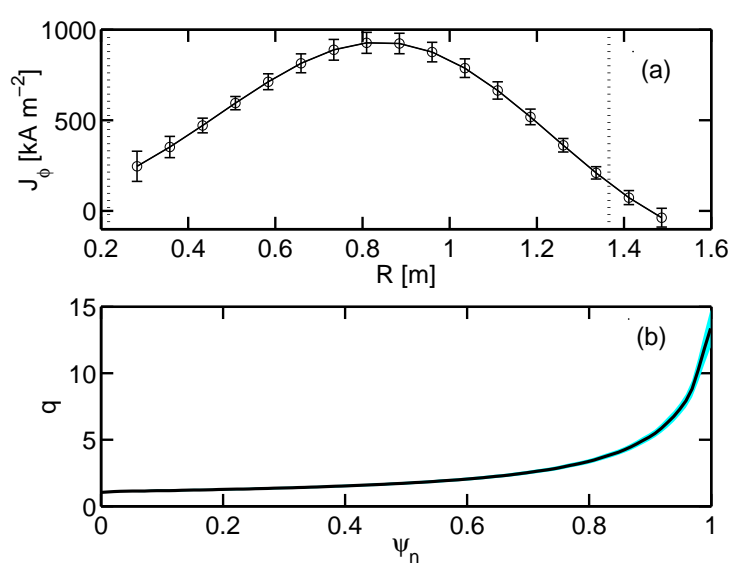

Figure 2. Profiles of (a) toroidal current $J_{\phi}$ and its standard deviation across the midplane and (b) the safety factor or $q$ profile as a function of normalized poloidal flux for shot \#20662 at 230ms. In (a) the dashed lines denote the inner and outer boundary of the plasma. The shaded area in (b) represents the uncertainty inferred from Bayesian analysis. Also in (b) the poloidal flux is normalized such that $\psi_{n}=0$ is the magnetic axis and $\psi_{n}=1$ is the edge. 
where $H$ is a flux function, $T$ is the single-fluid temperature, $m_{i}$ is ion mass, and $\rho_{0}$ is an arbitrary constant. Eliminating $v_{\phi}$ from Eqs. (2) and (3) we obtain the following expression for particle density $n \approx \rho / m_{i}$ :

$$
n=n_{1}(\psi) \exp \left[\frac{\Omega^{2}(\psi)}{2 v_{i}^{2}(\psi)}\left(R^{2}-R_{0}^{2}\right)-\frac{1}{2} M_{s \theta}^{2}\right],
$$

where $n_{1}$ is a flux function, $v_{i}=\left(2 T / m_{i}\right)^{1 / 2}$, and $R_{0}$ is an arbitrary constant. The quantities $\Omega, v_{i}$ and $M_{s \theta}=\left(v_{\theta} / v_{i}\right)\left(B / B_{\theta}\right)$ can be measured on the low field side of the plasma using neutral beam diagnostics (charge exchange and motional Stark effect). Because of beam attenuation and injection geometry, such measurements are generally not possible on the high field side.

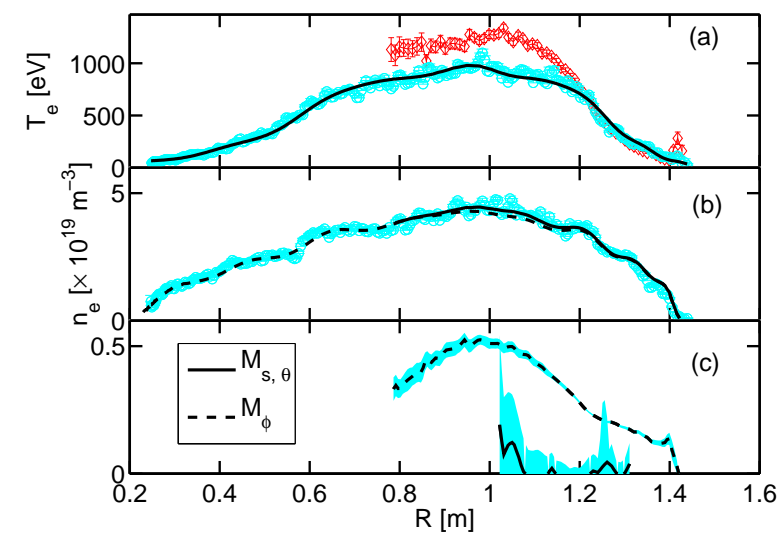

Figure 3. Profiles during the early L-mode phase of a 700kA discharge (\#20662 at $230 \mathrm{~ms}$ ) with $1.8 \mathrm{MW}$ beam heating. Shown are profiles of (a) electron temperature (blue circles), electron temperature fit (solid black line), and ion temperature (red diamonds), (b) electron density (blue circles), and (c) inferred poloidal and toroidal Mach numbers and their uncertainty (blue shading). In (a) and (b) the solid lines are fits as a function of radius, and the dashed line in (b) a fit for $n_{1}(\psi)$, which would be the density profile in the absence of toroidal rotation.

We have explored rotation induced asymmetry of plasma density and computed Mach numbers for MAST discharge \#20662 at $230 \mathrm{~ms}$, studied in Sec. 1. Figure 3 shows TS and Charge Exchange Recombination Spectroscopy (CXRS) data, together with inference of outboard measurements of toroidal rotation Mach number profile $M_{s, \phi}=v_{\phi} / v_{i}$ and poloidal rotation Mach number profile $M_{s, \theta}$. The CXRS $T_{i}$ data provides a measure of the ion thermal speed $v_{i}$. Next, we have used the EFIT computed $\psi$ profile and the $T_{e}$ profile to locate the magnetic axis at $R=0.95$ : this is found by linearly adjusting $\psi$ so as to minimise the difference between interpolated inboard and outboard electron temperature. Polynomial fits to $T_{e}$ and $n_{e}$ are based on this shifted profile. Except for differences in the magnetic axis and outer boundary, radial profiles of the normalised $\psi$ from EFIT and the expectation values of the MINERVA computed normalised $\psi$ map onto each other, and so using a mapped $\psi$ from EFIT is reasonable. Finally, in Fig. 3(b), the dashed line is a plot of $n_{1}(\psi)$, obtained by dividing $n$ by the exponent offset on the RHS of Eq. (4) with $R_{0}=0$. 
For this discharge, rotation velocity profiles were obtained by a careful analysis of Doppler shift of line emission excited by charge exchange between energetic injected beam atoms and the ionised impurities [27]. For $M_{s, \theta}$ the ratio $B / B_{\theta}$ and its uncertainty has been computed using the Bayesian inference code MINERVA, as described in Sec. 1. We have generated uncertainty estimates for $M_{s, \theta}$ by linearly combining errors in the rotation profiles and magnetic field ratio $B / B_{\theta}$. The large value of the poloidal Mach number on-axis $(\approx 0.2)$ occurs because of the local poloidal field null. As shown, the uncertainty in poloidal Mach number is comparable to the mean. The poloidal flow effects on the density profile are small because the square of the Mach number is small. Importantly, the poloidal Mach number, at least on the outboard side, is not large enough to pass through unity and thus generate a contact discontinuity of the type associated with a change in the Grad-ShafranovBernoulli solution domain from elliptic to hyperbolic [29] (it should be noted that such MHD discontinuities are generally replaced by smooth gradients when two-fluid [30] or kinetic effects [31] are taken into account).To our knowledge, these represent first results of the measured inference of both the poloidal Mach number and its uncertainty. In contrast, the uncertainty in toroidal Mach number is small, and the toroidal Mach number large $(\approx 0.5)$. The toroidal rotation-induced asymmetry evident in the density profile has been used elsewhere to infer the toroidal rotation profile. [32].

In this discharge $\Omega^{2} / v_{i}^{2}$ is sufficiently small that the term in the exponent in Eq. (4) that is proportional to this quantity causes little inboard/outboard asymmetry in the density. Significant asymmetry could in principle arise from the $M_{s \theta}$ term in the exponent, since this is proportional to $B / B_{\theta}$, which is generally much larger than unity on the high field side of a spherical tokamak plasma. Figure 3(b) indicates that there is in fact little asymmetry in the density profile, and hence we may conclude that in this case $M_{s \theta}$ is likely to be less than unity on the high field side as well as the low field side. However measurements of flows in other tokamaks, notably JET, show that $M_{s \theta}$ can be of order unity in the vicinity of internal transport barriers [10].

Using the last closed flux surface from the Bayesian MSE model, and the fitted density and temperature profiles and the toroidal rotation profile we have also computed rotating and stationary equilibria for \#20662 at $230 \mathrm{~ms}$ using the FLOW code [29]. The purpose of the calculation is to compute the effects of toroidal rotation on an experimental magnetic configuration using experimental pressure profiles as derived from Thomson scattering. The impact of these profiles on plasma stability, as compared to other static equilibria, has been addressed elsewhere. [1] FLOW is constrained by flux functions $D(\psi), P(\psi), M_{s, \theta}(\psi), M_{s, \phi}(\psi)$ and $B_{0}(\psi)$, representing a quasidensity, quasipressure, quasisonic poloidal Mach number, quasisonic toroidal Mach number, and quasitoroidal field, respectively. In the presence of toroidal flow these functions are not physical, and so care must be taken when constraining FLOW to discharges with rotation. We have taken $D(\psi)=n_{i} / n_{e} n_{1}(\psi)$ in Eq. (4), with $R_{0}$ chosen to be the geometric axis. For this discharge we have taken $n_{i} / n_{e}=0.8$, which is typical for MAST plasmas with the effective charge $Z_{\text {eff }}$ in the range $2<Z_{\text {eff }}<3$. [1] The quasipressure is constrained by $P(\psi)=k_{B} D(\psi) T(\psi)\left(1+\frac{n_{e}}{n_{i}} \frac{T_{e}}{T i}\right)$. The ratio $T_{i} / T_{e}=1.2$ is set by the ratio of core temperatures in Fig. 3(a). The toroidal Mach number profiles has been taken directly from Fig. $3(\mathrm{~b})$, and we have set $M_{s, \theta}=0$, which is correct to within error. In all cases we have computed $\psi$ profiles by interpolating radial profiles to $\psi$ profiles in Fig. 3 which use the TS corrected EFIT $\psi$. Finally, we have taken $B_{0}(\psi)$ from EFIT, because MINERVA uses the vacuum field.

Figure 4 shows the plasma reconstruction with toroidal flow. The magnetic 
axis for the reconstruction, as inferred from Fig. 4(a) and 4(b) lies at $R=0.95$, in agreement with fits to the $T_{e}$ data in Fig. 3(a). Overlaid on Fig. 4(c) is the mass density profile and its uncertainty inferred from TS $n_{e}$ data: these show reasonable agreement. While not shown here, the computed solution for $\rho(r)$ agrees with the fitted density profile $n_{1}(\psi)$ shown in Fig. 3(b) to within the discrepancy between the poloidal flux of MSE and the computed solution shown in Fig. 4(b). We have included the EFIT pressure profile and its gradient as a reference to the computed pressure profile and its gradient using TS inferred pressure as an input. Our purpose is to illustrate the effect of the change in pressure on the equilibrium, not provide a reference equilibrium with zero flow. The effect of replacing the pressure profile is to slightly change the $q$ profile from the EFIT solution. The effect is most notable for $\psi_{n}<0.05$, and the central safety factor drops to $q_{0}=0.40$ compared to the EFIT value of $q=1.04$, as shown in Fig. 4(g). Finally, we have included the toroidal flux profile for completeness.

We have also computed the solution in the absence of toroidal rotation, for which we expect $\rho(\psi)=m D(\psi)=n_{1}(\psi) n_{i} / n_{e}$. This is shown by the dashed line in Fig. $4(\mathrm{c})$. The impact of toroidal rotation in this discharge is to increase the radial position of the magnetic axis by $\approx 1 \%$, and lower the central safety factor by $\approx 5 \%$. Both are consistent with earlier findings by Hole and Dennis [12] for $M_{s, \phi}<0.5$.

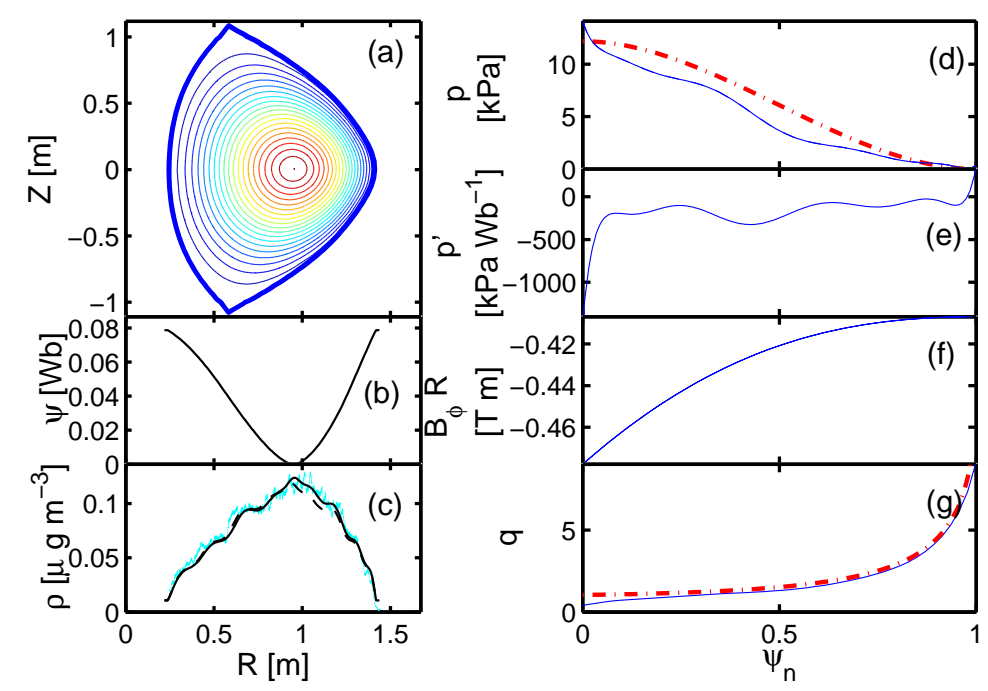

Figure 4. Reconstruction of \#20662 at 230 ms using last closed flux surface in Fig. 1, and profile information from Fig. 3. Part (a) shows a poloidal flux contour map, with input boundary profile overlaid (heavy line); (b) shows the poloidal flux across the midplane: (c) shows the mass density profile for the reconstruction with flow (solid) and no flow (dashed) across the midplane; (d) shows the computed pressure profile (solid) and EFIT pressure profile (dashed-dot); (e) shows the computed pressure gradient profile; (f) shows the computed toroidal flux function; and (g) shows the computed $q$ profile and the EFIT $q$-profile (dashed-dot). The shading in part (c) represents the region about one standard deviation of the mass density profile inferred from TS $n_{e}$ data. The profile information in parts (d)-(f) is given as a function of normalised poloidal flux $\psi_{n}$. 


\section{Anisotropy}

The inclusion of pressure anisotropy is also known to have an effect on a flowing equilibrium.[33] For instance, the characteristic poloidal flow speeds at which the Grad-Shafranov equation is hyperbolic is changed for large anisotropy. The centrifugal shift can also be increased or diminished/reversed with larger perpendicular or parallel temperature, respectively.

We have computed pressure anisotropy using the TRANSP [34] NBI fast ion distribution output for MAST \# 18696, a $700 \mathrm{kA}$ discharge with $1.9 \mathrm{MW}$ of neutral beam heating and a normalised beta of $\beta_{N}=2.5$. The discharge, acquired during an Alfvén Eigenmode campaign [35], was chosen for the finely converged TRANSP simulation [36].

Fast particle beam density, velocity and pressure can be computed as successive moments of the fast ion distribution $\hat{f}(E, \lambda)$, where $E$ is the particle energy and $\lambda$ is the cosine of the angle of the velocity from parallel. The required integrals are given by

$$
\begin{aligned}
& n=\int_{0}^{\infty} \int_{-1}^{1} \hat{f}(E, \lambda) d \lambda d E \\
& n u_{\|}=\int_{0}^{\infty} \int_{-1}^{1} v_{\|} \hat{f}(E, \lambda) d \lambda d E \\
& p_{\|}=m \int_{0}^{\infty} \int_{-1}^{1}\left(v_{\|}-u_{\|}\right)^{2} \hat{f}(E, \lambda) d \lambda d E \\
& p_{\perp}=\frac{m}{2} \int_{0}^{\infty} \int_{-1}^{1} v_{\perp}^{2} \hat{f}(E, \lambda) d \lambda d E
\end{aligned}
$$

Figure 5 shows fast ion density and parallel mean flow speed as well as the computed parallel and perpendicular pressures. For this discharge, a ratio $p_{\perp} / p_{\|} \approx 1.7$ was found.
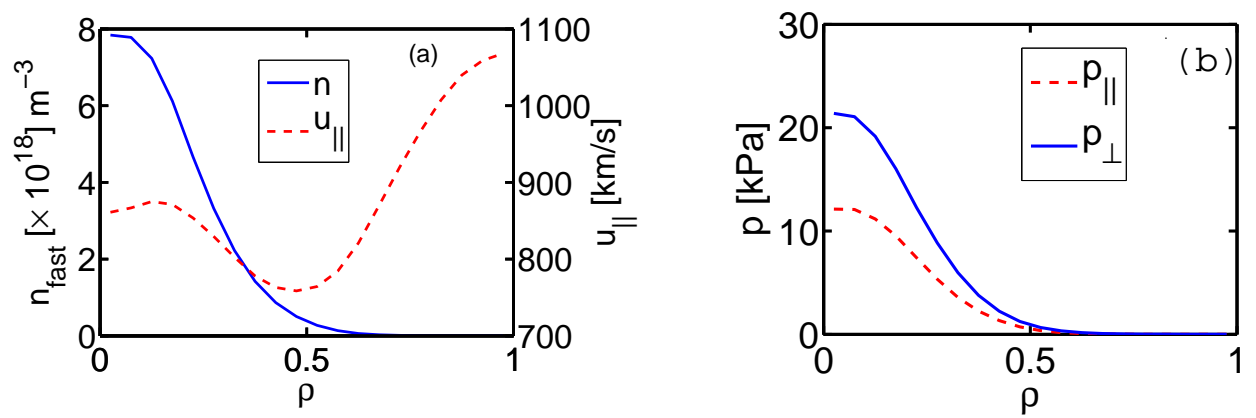

Figure 5. Profiles of (a) fast ion density and parallel mean flow speed and (b) parallel and perpendicular pressures, computed from a TRANSP distribution function for MAST discharge \#18696. The horizontal axis is the standard flux label coordinate $\rho=\sqrt{\Phi / \Phi_{0}}$ where $\Phi$ is the toroidal flux enclosed within a given flux surface and $\Phi_{0}$ is the corresponding value at the boundary. 
Upper-limit changes to the isotropic and static equilibrium implied by these values were calculated using FLOW [29]. The density, pressure and velocity moments of the NUBEAM fast ion distribution function were used in calculations of the anisotropy, $\Delta=\mu_{0}\left(p_{\|}-p_{\perp}\right) / B^{2}$, and toroidal Alfvénic Mach number $M_{A, \phi}=v_{\theta} / c_{A}$, with $c_{A}=\sqrt{\mu_{0} \rho} / B$ the Alfvén speed. Poloidal flow was neglected, and the toroidal velocity magnitude was taken to be the magnitude of the velocity moment.

The free functions $p(\psi)$ and $f(\psi)$ and were taken from a time-slice of the TRANSP run \#18696B0 at 0.290s. The energy of each isotropic degree of freedom was redistributed between $p_{\perp}$ and $p_{\|}$in the anisotropic cases to give the required ratio of 1.7. It is important to note that the usual expression $f(\psi)=B_{\phi} R / \mu_{0}$ is incorrect for the case of anisotropy or flow and care must be taken when using the toroidal field to calculate $f(\psi)[29]$.
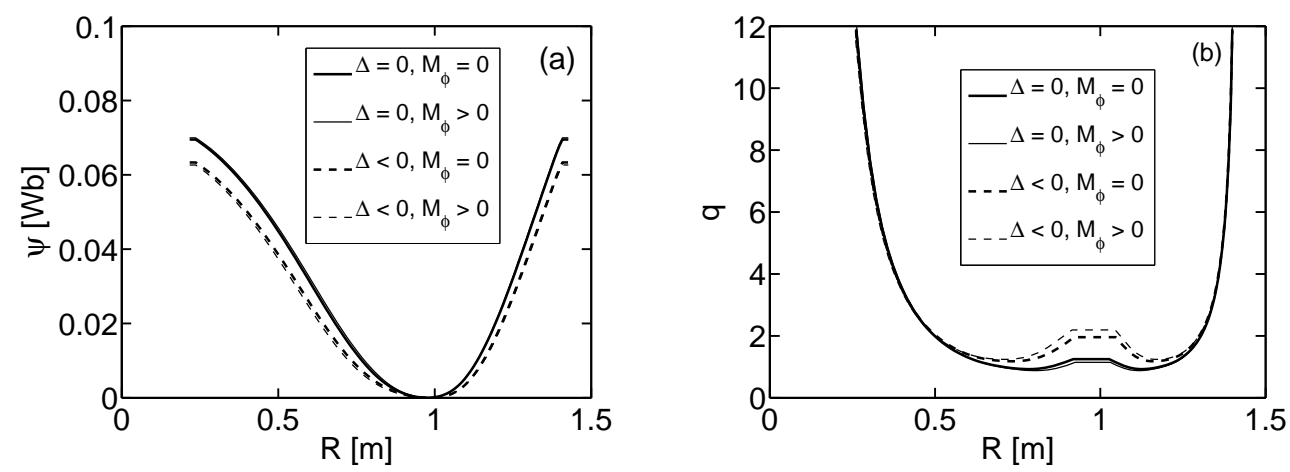

Figure 6. Profiles of (a) poloidal flux and (b) q as computed using FLOW consistent with anisotropy and rotation calculated from TRANSP for MAST discharge $\# 18696$. Here, $\Delta<0$ corresponds to an anisotropy of $p_{\perp} / p_{\|} \approx 1.7$, and $M_{A, \phi}>0$ corresponds to a nonzero toroidal flow profile with a maximum Alfvénic Mach number of 0.3 .

Figure 6 consists of the results of the FLOW computation including rotation and anisotropy, using the guided centre model closure [37,33]. It is evident that the toroidal rotation $\left(M_{A, \phi} \leq 0.3\right)$ did not change the equilibrium appreciably, however the pressure anisotropy reduced the poloidal flux by $15 \%$ where the pressure gradient was greatest. A corresponding decrease in $B_{\theta}$ resulted in an increase in $q$ on axis.

We conclude that anisotropy introduced by the presence of energetic particle populations can be significant in determining the plasma equilibrium. In particular, it is known [33] that for systems with pressure anisotropy of $p_{\perp}>p_{\|}$, an increase will occur in centrifugal shift of the density profile.

\section{Conclusions}

We have reported on several advances in equilibrium modelling and interpretation. The focus of the work is on measuring and quantifying the impact of rotation and anisotropy. We have folded rotation profiles, CXRS data, TS data and Bayesian inferred magnetic configuration information together to compute poloidal and toroidal Mach numbers. These show that MAST plasmas are near stationary in the poloidal 
direction, with a poloidal Mach number of zero within uncertainty. The toroidal rotation can however be significant in MAST plasmas: we compute a toroidal Mach number of 0.5 on-axis. Using the fitted profile information we have computed the equilibria for both toroidally flowing and stationary plasmas. This shows that the effect of toroidal rotation is small, acting to increase the magnetic axis by $\approx 1 \%$, and lower the central safety factor by $\approx 5 \%$. In comparison, corrections to make the pressure profile consistent with internal measurements such as CXRS and TS have a much larger impact. A separate approach to computing the magnetic configuration is afforded by Bayesian inference. In this case MSE, flux loops, and pick up coils are folded into a probablistic framework to enable extraction of the poloidal flux, safety factor, current density, and their uncertainty. The uncertainty is dominated by the undetermined nature of the inference and the errors associated with each diagnostic observation.

In other work we have computed the magnitude of anisotropy for a MAST discharge using a TRANSP NBI distribution function. The impact of this anisotropy $p_{\perp} / p_{\|} \approx 1.7$ is to lower the poloidal field $B_{\theta}$, and hence boost the safety factor. In ongoing work we are implementing rotation and anisotropy into the Bayesian inference engine MINERVA, so as to compute plasma configurations in beam-heated highperformance plasmas, and determine the uncertainty in inference of the configuration. In future we also plan to compute the impact of anisotropy on plasma stability.

\section{Acknowledgments}

The authors would like to thank Dr Anthony Field and Dr John McCone of the Culham Centre for Fusion Energy for advice on data for MAST discharge \#20662, and Dr Robert Budny of Princeton Plasma Physics Laboratory for provision of a TRANSP simulation for MAST discharge \#18696. This work was partly funded by the Australian Government through Australian Research Council grants FT19899 and DP10993797 and International Science Linkages Grant CG130047, the Australian National University, the RCUK Energy Programme, and by the European Communities under the contract of Association between EURATOM and the Culham Centre for Fusion Energy.

\section{References}

[1] M.J. Hole, R.J. Akers, L.C. Appel, R.J. Buttery, C. Brickley, N.J. Conway, M. Gryaznevich, T.C. Hender, O.J. Kwon, M. Valovic, S. Medvedev, A. Patel, S. Saarelma, D. Taylor, H.R. Wilson, and the MAST Team. Plasma Phys. Controlled Fusion, 47:581-613, 2005.

[2] F. L. Hinton and S. K. Wong. Phys. Fluids, 28:3082-3098, 1985.

[3] P. Helander. Phys. Plas., 5:1209-1211, 1998.

[4] K. G. McClements and R. J. McKay. Plas. Phys. Con. Fus., 51(115009), 2009.

[5] M. Artun and W. M. Tang. Phys. Fluids B, 4:1102-1114, 1992.

[6] K. H. Burrell. Phys. Plas., 4(5):1499-1518, 1997.

[7] I.T. Chapman, T.C. Hender, S. Saarelma, S.E. Sharapov, R.J. Akers, N..J. Conway, and the MAST Team. Nuc. Fus., 46:1009-1016, 2006.

[8] Yueqiang Liu, M. S. Chu, A. M. Garofalo, R. J. La Haye, Y. Gribov, M. Gryaznevich, T. C. Hender, D. F. Howell, P. de Vries, M. Okabayashi, S. D. Pinches, H. Reimerdes, and EFDAJET contributors. Phys. Plas., 13:056120, 2006.

[9] K. Crombé, Y. Andrew, M. Brix, C. Giroud, S. Hacquin, N. C. Hawkes, A. Murari, M. F. F. Nave, J. Ongena, V. Parail, G. Van Oost, I. Voitsekhovitch, and K.-D. Zastrow. Phys. Rev. Lett., 95:155003, 2005.

[10] K. G. McClements and M. J. Hole. Phys. Plas., 17:082509, 2010. 
[11] M. J. Hole and K. G. McClements. J. Phys. Conf. Ser., 260(012013), 2010.

[12] M. J. Hole and G. Dennis. Plas. Phys. Con. Fus., 51:035014, 2009.

[13] G. A. Cottrell. chapter Maximum entropy and plasma physics. Oxford Science, Oxford, 1990.

[14] A. P. Millar, D. C. McDonald, and D. A. Diver. Plas. Phys. Cont. Fus., 42:337-346, 2000.

[15] R Fischer, A Dinklage, and E Pasch. Plas. Phys. Cont. Fus., 45:1095-1111, 2003.

[16] A Dinklage, R Fischer, and J Svensson. Fus. Sci. Techn., 46:355-364, 2004.

[17] J Svensson, A Dinklage, J Geiger, A Werner, and R Fischer. Rev. Sci. Instrum., 75(10):42194221, Jan 2004.

[18] J. Svensson and A. Werner. Plas. Phys. Cont. Fus., 50:085002, Jan 2008.

[19] Oliver Ford, J. Svensson, A. Boboc, D. C. McDonald, and JET-EFDA Contributors. Rev. Sci. Instrum., 79(10):10F324, 2008.

[20] M. Reginatto and A. Zimbal. Rev. Sci. Instrum., 79:023505, 2008.

[21] J Svensson and A Werner. IEEE International Symposium on Intelligent Signal Processing, pages 1-6, Jan 2007.

[22] M. J. Hole, G. von Nessi, J. Bertram, J. Svensson, L. C. Appel, B. D. Blackwell, R. L. Dewar, and J. Howard. Journal of Plasma and Fusion Research, 9, 2010.

[23] M. J. Hole, G. von Nessi, D. Pretty, J. Howard, B. Blackwell, J. Svensson, and L. C. Appel. Rev. Sci. Instrum., 2010.

[24] G. T. von Nessi, M. J. Hole, J. Svensson, and L. Appel. Plas. Phys. Con. Fus., 2011. submitted $07 / 09 / 2010$.

[25] H. Meyer and et al. Nuc. Fus., 49(10):104017, 2009.

[26] M. J. Hole, L. C. Appel, and R. Martin. Rev. Sci. Instrum., 80:123507, 2009.

[27] A. R. Field, J. McCone, N. J. Conway, M. Dunstan, S. Newton, and M. Wisse. Plas. Phys. Con. Fus., 51:105002, 2009.

[28] K. G. McClements and A. Thyagaraja. Mon. Not. R. Astron. Soc., 323:733-742, 2001.

[29] L. Guazzotto, R. Betti, J. Manickam, and S. Kaye. Phys. Plasmas, 11(2):604-614, February 2004.

[30] J. P. Goedbloed. Phys. Plasmas, 11:L81-L84, 2004.

[31] A. Bondeson and R. Iacono. Phys. Fluids B, 1(7):1431-1443, 1989.

[32] R. J. Akers et al. Plas. Phys. Con. Fus., 45:A175-A204, 2003.

[33] R. Iacono, A. Bonderson, F. Troyon, and R. Gruber. Phys. Fluids B, 2(8):1794-1803, 1990.

[34] R. J. Hawryluk. In B. Coppi, editor, Physics of plamas close to thermonuclear conditions, volume 1, pages 19-46, CEC Brussels, 1980.

[35] M. P. Gryaznevich, S. E. Sharapov, M. Lilley, S. D. Pinches, A. R. Field, D. Howell, D. Keeling, R. Martin, H. Meyer, H. Smoth, R. Vann, P. Denner, E. Verwichte, and the MAST Team. Nuc. Fus., 48(084003), 2008.

[36] M. K. Lilley, S. E. Sharapov, H. M. Smith, R. J. Akers, D. McCune, and the MAST team. volume 32D, Hersonissos, June 2008.

[37] D. Dobrott and J. M. Greene. Phys. Fluids, 13:2391-2397, 1970. 